\title{
SECURITY CONSTRAINED OPTIMAL LOAD DISPATCH USING HPSO TECHNIQUE FOR THERMAL SCHEDULING PROBLEMS
}

\author{
S.Prabakaran ${ }^{1}$, V.Senthil Kumar ${ }^{2}$ \\ ${ }^{I}$ Research Scholar, ${ }^{2}$ Associate Professor, Department of Electrical and Electronics Engineering, Anna University, \\ Chennai-600025, Tamilnadu, India \\ prabakaran110768@gmail.com
}

\begin{abstract}
This paper presents Hybrid Particle Swarm Optimization (HPSO) technique to solve the Optimal Load Dispatch (OLD) problems with line flow constrain, bus voltage limits and generator operating constraints. In the proposed HPSO method both features of EP and PSO are incorporated, so the combined HPSO algorithm may become more effective to find the optimal solutions. In this paper, the proposed Hybrid PSO, PSO and EP techniques have been tested on IEEE14, 30 bus systems. Numerical simulation results show that the Hybrid PSO algorithm outperformed standard PSO algorithm and Evolution Programming method on the same problem and can save considerable cost of Optimal Load Dispatch.
\end{abstract}

Keywords: Evolutionary Programming, Gaussian Mutation, Particle Swarm Optimization, Hybrid PSO, Optimal Load Dispatch, Line Flow Constraints

\section{INTRODUCTION:}

Optimal Load Dispatch (OLD) pertains to optimum generation in an interconnected power system to minimize the cost of generation subject to relevant system constraints. In this paper the line loading (MVA) and voltage constraints, important for any practical implementation of short term OLD, are taken into consideration. The control of voltages, real and reactive power limit (MVA limit) on the transmission line is one of the most important activities in the modern power system. In the past, many mathematical programming models and optimization technique have been applied to solve the OLD problems. These methods include lambda iteration method [1], base point, participation factor, gradient method, etc. However, the base case operating constraints, line flow limits and load bus voltage magnitude limits are not consider in this methods. Ringlee et al.[2] to solved a non-convex OLD problem using Dynamic Programming (DP) but this has disadvantage namely the computational requirements of the DP based method depend on the size of the discrete capacity step (10MW, 20MW) used, Which is usual accuracy required in the OLD schedule. Dommel et al [3] presented a NonLinear Programming (NLP) technique to solve Optimal Power Flow (OPF) problem in which the line flow constraints and voltage limits are included. Nanda et al [4] have developed an algorithm to solve the OLD problem with line flow constraints using modified coordination equations.

Linear programming methods are fast and reliable, but the main disadvantage is the piece-wise linear cost approximation.
NLP methods have a problem of convergence and algorithm complexity.

Stochastic searching algorithms such as Simulate Annealing (SA) [5] and Hopfield neural network methods [6] have also been used to solve the non-convex OLD problem. However, these methods require external training routines. Baskar et al [7] proposed a participation factor in conjunction with the improved lambda based algorithm (GA) to solve OLD problem but this has disadvantage that the line flow limits are not considered and it leads to overload on the lines. Though Meta heuristic algorithm such as GA has been employed to solve OLD problems, recent research has identified some deficient in GA performance. The premature convergence of GA degrades its performance and reduces its search capability that leads to a higher probability towards obtaining local minimum. The main objective of the present work is to develop and study the absolute as well as relative performance of following techniques applied to the power system OLD problem with line flow constraints, voltage on each bus, minimum and maximum generating limits and power balance constraint. The PSO Algorithm was applied to solve various optimal load dispatch problems [10],[11],[12]. The security constraints OLD Problem is solved and necessary software has been developed using the following techniques:

1. Evolutionary Programming (EP) [8]

2. Particle Swarm Optimization (PSO) [9]

3. Proposed Hybrid PSO 


\section{PROBLEM FORMULATION:}

Optimization of fuel cost for generation has been formulated based on a classical OLD problem with line flow constraints. For a given power system network, the optimized cost of generation is given by the following equation,

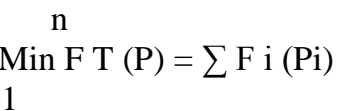

Subject to

(i) Power balance equation

n

$\sum \operatorname{Pgi}=\underset{i=1}{\mathrm{Pd}}+\mathrm{Pl}$

(ii) The power flow equation of power network

$\mathrm{g}(|\mathrm{v}|, \theta)=0$

Where

$$
g(|v|, \theta)=\mid \begin{aligned}
& \text { Pi }(|v|, \theta)-\text { Pinet } \\
& \text { Qi }(|v|, \theta)-\text { Qinet } \\
& \text { Pm }(|v|, \theta)-\text { Pinet }
\end{aligned}
$$

(iii) The inequality constraint on real power generation Pgi of each unit $\mathrm{i}$,

$$
\text { Pgimin } \leq \text { Pgi } \leq \text { Pgimax }
$$

(iv) The inequality constraint on voltage of each PQ bus Vimin $\leq$ Vi $\leq$ Vimax

(v) Power limit on transmission line MVAfp,q $\leq$ MVAfp,qmax

Total fuel cost of generation FT in terms of control variables generator power can be expressed as

$$
\mathrm{F}(\mathrm{Pi})=\sum(\underset{\mathrm{aiPgi} 2}{\mathrm{n}}+\mathrm{bi} \mathrm{Pgi}+\mathrm{ci}) \quad \$ / \mathrm{hr}
$$

\section{OVERVIEW OF EP AND PSO:}

Four decades earlier EP was proposed for evolution of finite state machines, in order to solve a prediction task. Since then, several modifications, enhancements, and implementations have been proposed and investigated. Mutation is often implemented by adding a random number or a vector from a certain distribution (e.g., a Gaussian distribution in the case of EP to a parent). The degree of variation of Gaussian mutation is controlled by its standard deviation, which is also known as a 'strategy parameter' in an evolutionary search.

PSO is a population based optimization method first proposed by Kennedy and Eberhart. According to the background of PSO and simulation of swarm of bird, Kennedy and Eberhart [10] [11] developed a PSO concept.

PSO is basically developed through simulation of bird flocking in two- dimensional space. The position of each agent is represented by $\mathrm{XY}$ axis position and also the velocity is expressed by Vx (velocity of $\mathrm{X}$ axis) and Vy (velocity of $\mathrm{Y}$ axis). Modification of the agent (particle) position is realized by the position and velocity information. Bird flock- ing optimizes a certain objective function. Each agent knows its best value so far (pbest) and its XY position. This information is analogy of personal experiences of each agent. Moreover, each agent knows the best value so far in the group (gbest) among pbests. This information is analogy of knowledge of how other agents around them have performed. Each agent tries to modify its position using the following information:

- The current position (x,y),

- The current velocities (Vx, Vy),

- The distance between the current position and pbest,

- The distance between current position and gbest.

This modification can be represented by the concept of velocity. Velocity of each agent can be modified by the following equation

$\mathrm{Vit}+1=\mathrm{W}$ Vit $+\mathrm{C} 1 *$ rand $1 *($ pbest $\mathrm{i}-\mathrm{sit})+\mathrm{C} 2 *$ rand $2 *($ gbest sik0) (8)

The following weighing function is usually utilized in eqn (8)

$$
W=W_{\max }-\left(W_{\max }-\frac{\left.W_{\min }\right) * \text { iter }}{\text { iter }_{\max }}\right.
$$

Using the above equation, a certain velocity, which gradually gets close to pbest and gbest can be Calculated. The current position can be modified by the following equation

Sit $+1=\operatorname{Sit}+\operatorname{Vi}(\mathrm{t}+1)$

The first term of the right hand side of (8) is corresponding to diversification in the search procedure. The second and third terms of that are corresponding to intensification in the search procedure.

The PSO method has a well-balanced mechanism to utilize diversification and intensification in the search procedure efficiently. Figure 1 shows the concept of modification of a searching point by PSO. Figure 2 shows the general flowchart of PSO method.

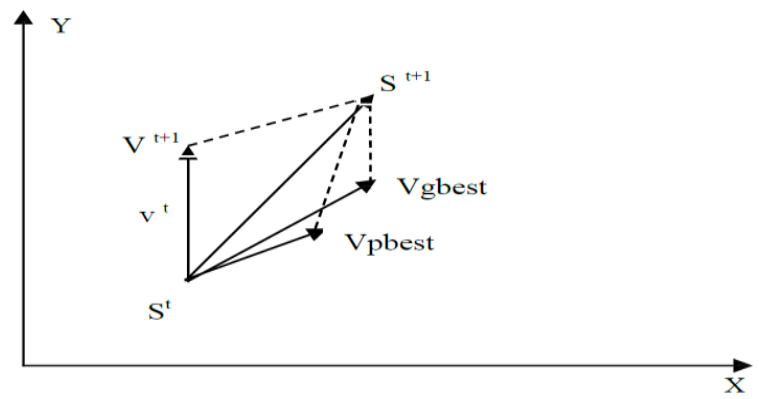

Fig 1: Concept of modification of a searching point by PSO 
$S^{t} \quad$ Current searching point

$\mathrm{S}^{\mathrm{t}+1}$

$\mathrm{V}^{\mathrm{k}}$

$\mathrm{V}^{\mathrm{k}+1}$

Vpbest

Vgbest

Velocity based on pbest

Velocity based on gbest
Modified searching point

Current velocity

Modified velocity

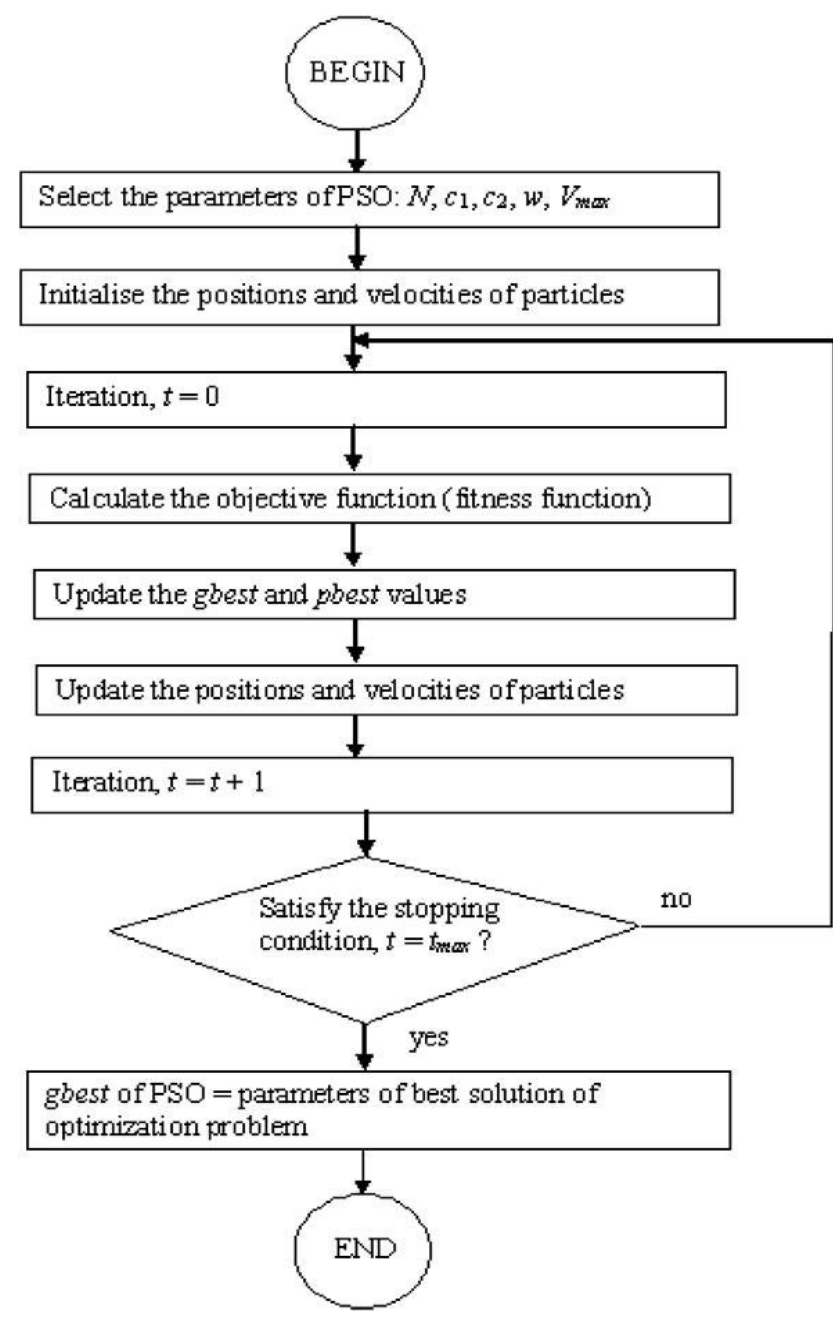

Fig2. Flow Chart of General PSO Method

\section{DEVELOPMENT OF HYBRID PSO METHOD}

Using the above concepts, the Hybrid PSO (PSO Combined EP) algorithm can be expressed as given below. Initial searching points (real power generation of generators) and velocities are usually generated randomly within allowable range. The current searching point is set to pbest for each agent. The best evaluated value (minimum augmented fuel cost value in OLD problem) of pbest is set to gbest and gbest value stored.
Modification of searching point of each agent is changed using (8), (9) and (10) and the evaluation values are calculated (augmented fuel cost value in OLD problem). If the evaluation value of each agent is better than the previous pbest, the value is set to pbest. If the best pbest is better than previous gbest, the value is set to gbest. Modification of searching points using gaussian random variable with 0 mean and standard deviation proportional to scaled cost values (EP method) and the evaluation values are calculated. If the evaluation value of each agent is better than the previous pbest, the value is set to pbest. If the best pbest is better than previous gbest, the value is set to gbest. Modification of searching points using cauchy random variable (EP method) and the evaluation values are calculated. Figure 3 shows the flowchart of proposed HPSO method.

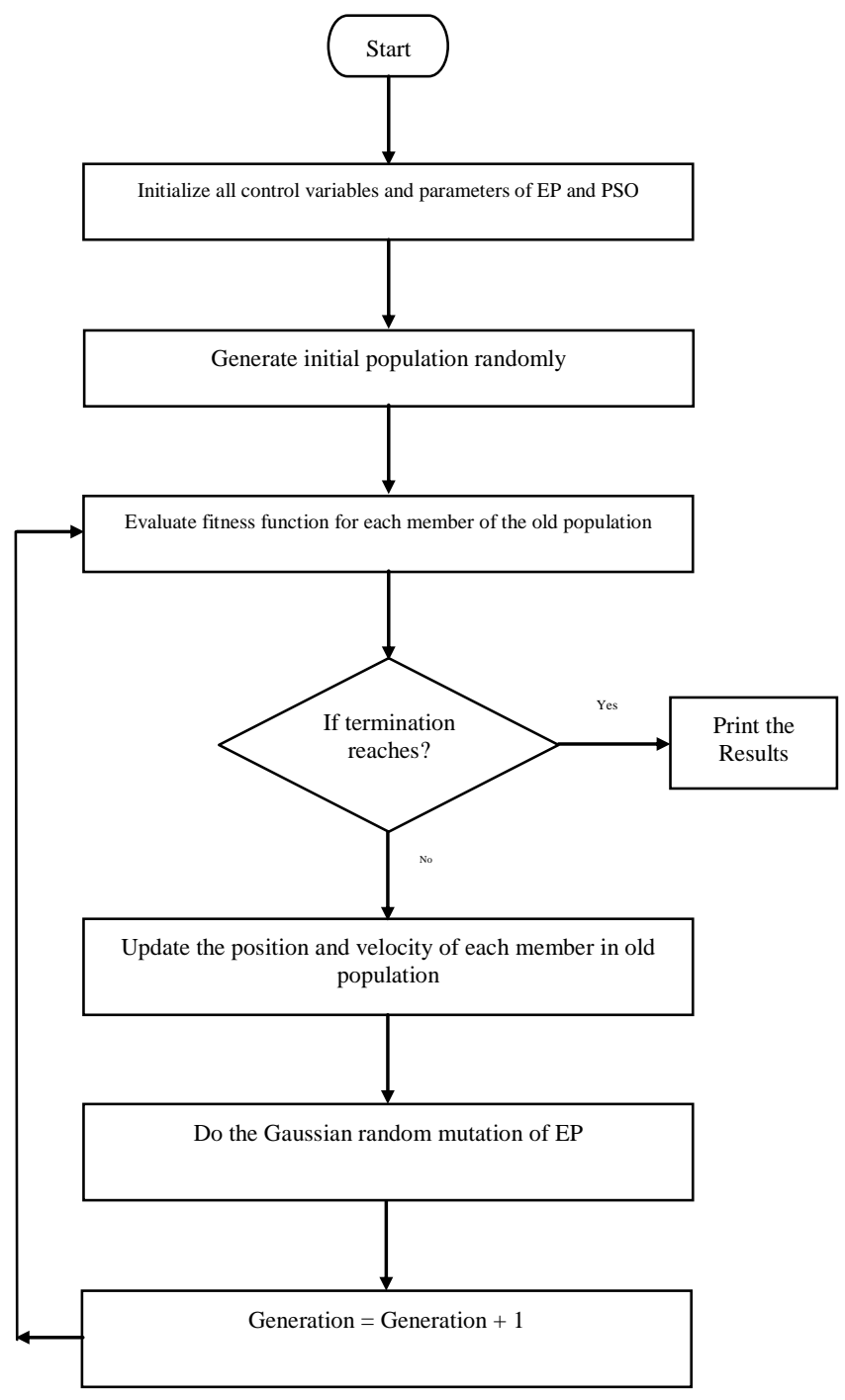

Fig.3. Flow Chart of HPSO methods 


\subsection{Step-by-step Procedure of Proposed Hybrid PSO}

\section{Method with Line Flow Constraints}

The search procedure of the proposed Hybrid PSO method for OLD problems with Line flow constraints is given below.

Step 1: Initialize randomly the real power generation $\mathrm{Pg} \mathrm{i}$ of the population according to the limit of each unit (except slack bus) including the individual dimensions, searching points and velocities. Initial velocity limits of each individual are as

$$
V_{d}^{\max }=0.5 P_{d}^{\max }, \quad V_{d}^{\min }=-0.5 P_{d}^{\text {min }}
$$

Where, $\quad P_{d}^{M I N}=\sum_{\mathrm{i}=1}^{\mathrm{n}} P_{t}^{M I N} \quad P_{d}^{M I N}=\sum_{\mathrm{i}=1}^{\mathrm{n}} P_{t}^{\max }$

Step 2: Compute slack bus generator vector (Ps), losses and line flows using New- ton-Raphson load flow method for the above generators.

Step 3: To account for slack unit limit violation, branch power flow limit violation and voltage limit violation, the total operating cost is augmented by non-negative penalty terms K1, K2 and K3.Augmented cost FT calculated using (11).

$$
F_{T}^{*}=F_{T}+K_{1}{ }^{*} \sum_{i=1}^{n l}\left(I_{i}-I_{i}^{\max }\right)^{2}+K_{2}{ }^{*}\left(P_{G 1}-P_{G 1}^{\mathrm{im}}\right)^{2}+K_{3} \sum_{i=1}^{N}\left(V_{L i}-V_{L i}^{\lim )^{2}}\right.
$$

Step 4: The minimum augmented fuel cost value among the population is taken as best value. The best augmented fuel cost value in the population is denoted as gbest and remaining individuals are assigned as pbest.

Step 5: Modify the member velocity $\mathrm{V}$ of the each individual Pgi using (12)

$v_{i d}^{i+1}=w^{*} v_{i d}^{(t)}+c_{1}^{*}$ rand $0 *\left(\right.$ pbest $\left._{i d}-p g_{i d}\right)+c_{2}^{*}$ Rand $^{*} *\left(\right.$ gbest $\left._{d}-p g_{i d}\right)$

where ' $n$ ' is the population size; ' $m$ ' is the number of units and the ' $w$ ' value is set using (9).

Step 6:

$$
\begin{aligned}
& \text { If } \quad V_{i d}^{(t+1)}>V_{d}^{\max } \text {, then } V_{i d}^{(t+1)}=V_{d}^{\max } \\
& \text { If } \quad V_{i d}^{(t+1)}<V_{d}^{\min } \text {, then } V_{i d}^{(t+1)}=V_{d}^{\min }
\end{aligned}
$$

Step 7: Modify member position of each individual Pgi using equation (13),

$$
P g_{d t}^{(t+1)}=P g_{d t}^{(n)}+V_{d i}^{(t+1)}
$$

Step 8: $P g_{i d}{ }^{(t+1)}$ Must satisfy the capacity limits of the generator as in (14)

$$
\begin{aligned}
& \text { If } P g_{u}^{(i+1)}>P g_{u}^{\text {mix }} \text { then, } P g_{u}^{(l+1)}=P g_{u}^{\operatorname{mix}} \\
& \text { If } P g_{u}^{(l+1)}<P g_{u}^{-} \text {then, } P g_{u}^{(i+1)=} P g_{u}^{\text {mi }}
\end{aligned}
$$

Step 9: Modified member positions in Step 8 are taken as initial value for $\mathrm{N}-\mathrm{R}$ load flow method. Compute slack bus power loss and line flows using N-R load flow method.

Step 10: Calculate the augmented fuel cost using equation (11). Assign gbest and pbest value. If the current gbest value is better than gbest value in Step 4 current value is set to gbest. If current pbest value is better than pbest value in Step 4 current value is set to pbest.

Step 11: $P g_{i d}{ }^{(t+1)}$ is created using Gaussian mutation as in (15) and (16).

$$
\begin{aligned}
& P g_{t d}^{(i+1))^{\prime}}=P g_{i d}+\mathrm{N}_{\mathrm{i}}\left(0, \sigma_{\mathrm{i}}\right) \\
& \sigma_{\mathrm{i}}=\beta^{*} \frac{f i}{f i \min }(\text { Pgimax }- \text { Pgimin })
\end{aligned}
$$

Check capacity limits of the generating units using (14), replacing $P g_{i d}{ }^{(t+1)}$ by $P g_{i d}{ }^{(t+1)^{\prime}}$

Step 12: Modified member positions in Step 11 are taken as initial value for $\mathrm{N}-\mathrm{R}$ load flow method. Compute slack bus power loss and line flows using N-R load flow method.

Step 13: Calculate the augmented fuel cost using (11). Assign gbest and pbest value. If the current gbest value is better than gbest value in Step 10 current value is set to gbest. If current pbest value is better than pbest value in Step 10 current value is set to pbest.

Step 14: If the iteration reaches the Maximum go to Step 15, otherwise go to Step 4, the gbest and pbest values in Step 4 replaced by latest gbest and pbest values from Step 13 .

Step 15: Individual that generates the latest gbest value is the optimal generation of each unit with minimum fuel cost satisfying all the Line flow constraints.

\section{EXAMPLES AND DISCUSSION:}

A comparative study of EP, PSO and Hybrid PSO methods were performed on IEEE 14 and 30 bus systems. The upper and lower voltage limits at all the buses except slack bus were taken as 1.01 and 0.95 respectively; the slack bus voltage was fixed to its specified of 1.06 PU. The line flows were computed using Newton-Raphson Method. Software has been developed in MATLAB to solve OLD problems using EP, PSO and Hybrid PSO methods and tested on Core i5 $3.0 \mathrm{GHz}$ 4GB RAM 500GB HDD Capacity personal Computer. Cost coefficient taken from [12] for IEEE 14 and 32 bus systems for implementing EP technique and PSO technique population 
size $=20$, maximum number of generations $=100$, is taken and the optimal solution was obtained in 50 trails

\section{Case Study 1: IEEE 14-Bus System}

The summarized results of IEEE 14 bus system are given in table 1 provides of EP results obtained by various optimization methods and the complete line flow results with and without line flow constraints using Hybrid PSO given in table 2. The star marked line was over loaded with economic generation scheduled when the line flow constrains are not considered. For IEEE14 bus system [12] demand of 259MW is taken. The results clearly show that the Hybrid PSO method is superior over the conventional PSO and EP methods.

Table1: Summary of Results 14 Bus system with Line Flow Constraints

\begin{tabular}{|c|c|c|c|c|c|}
\hline Method & $\begin{array}{c}\text { P1 } \\
(\mathrm{MW})\end{array}$ & $\begin{array}{c}\text { P2 } \\
(\mathrm{MW})\end{array}$ & $\begin{array}{c}\text { P3 } \\
(\mathrm{MW})\end{array}$ & $\begin{array}{c}\text { Losses } \\
(\mathrm{MW})\end{array}$ & $\begin{array}{c}\text { Optimum } \\
\text { Fuel Cost } \\
\$ / \mathrm{hr}\end{array}$ \\
\hline EP & 92.72 & 78.90 & 94.42 & 7.06 & 1103.9 \\
\hline PSO & 88.14 & 89.07 & 88.72 & 6.93 & 1114.9 \\
\hline $\begin{array}{c}\text { Hybrid } \\
\text { PSO }\end{array}$ & 114.44 & 52.18 & 100.00 & 7.62 & 1091.2 \\
\hline
\end{tabular}

Table2: Line Flow Results of Hybrid PSO IEEE 14-bus

\begin{tabular}{|c|c|c|c|c|}
\hline $\begin{array}{c}\text { Line } \\
\text { Designation }\end{array}$ & $\begin{array}{c}\text { Base } \\
\text { case } \\
\text { Line } \\
\text { Flow } \\
\text { in pu }\end{array}$ & $\begin{array}{c}\text { Line flow } \\
\text { with } \\
\text { Constraints } \\
\text { in pu }\end{array}$ & $\begin{array}{c}\text { Line Flow } \\
\text { Without } \\
\text { Constraints } \\
\text { in pu }\end{array}$ & $\begin{array}{c}\text { Max.line } \\
\text { Flow in } \\
\text { pu }\end{array}$ \\
\hline $1-2$ & 1.5741 & 0.5124 & $1.1025^{* *}$ & 1.0000 \\
\hline $1-5$ & 0.7485 & 0.3265 & 0.5014 & 1.0000 \\
\hline $2-3$ & 0.7312 & 0.6321 & 0.6124 & 1.0000 \\
\hline $2-4$ & 0.5142 & 0.3974 & 0.4215 & 0.5000 \\
\hline $2-5$ & 0.4258 & 0.2581 & 0.2146 & 0.5000 \\
\hline $3-4$ & 0.2145 & 0.3145 & 0.3698 & 0.5000 \\
\hline $4-5$ & 0.6589 & 0.6587 & 0.6123 & 1.0000 \\
\hline $4-7$ & 0.2985 & 0.1478 & 0.0968 & 1.0000 \\
\hline $4-9$ & 0.1859 & 0.0125 & 0.0478 & 0.5000 \\
\hline $5-6$ & 0.4125 & 0.2698 & 0.1254 & 0.5000 \\
\hline $6-11$ & 0.8965 & 0.2875 & 0.1632 & 0.5000 \\
\hline $6-12$ & 0.0125 & 0.1487 & 0.0968 & 1.0000 \\
\hline $6-13$ & 0.1547 & 0.2154 & 0.2365 & 1.0000 \\
\hline $7-8$ & 0.1985 & 0.1854 & 0.1587 & 0.5000 \\
\hline $7-9$ & 0.2968 & 0.1758 & 0.1968 & 0.5000 \\
\hline $9-10$ & 0.0321 & 0.1548 & 0.1245 & 0.5000 \\
\hline $9-14$ & 0.0214 & 0.0251 & 0.0621 & 0.5000 \\
\hline $10-11$ & 0.0145 & 0.0362 & 0.0141 & 1.0000 \\
\hline $2-13$ & 0.0245 & 0.0369 & 0.1544 & 1.0000 \\
\hline $13-14$ & 0.0241 & 0.1634 & 0.1457 & 0.5000 \\
\hline-19 & & & \\
\hline
\end{tabular}

** - Line Violation

\section{Case Study 2: IEEE 30-BUS System}

The summarized results of IEEE 30 bus system given in Table 3 provides of EP results obtained by various optimization methods. For IEEE 30 bus system demand of $283.4 \mathrm{MW}$ is taken. Line flow limits, bus voltage limits, capacity limit to consideration. The results clearly show that the proposed HPSO out performs the other methods.

Table3: Summary of IEEE 30 bus system with line flow constraints

\begin{tabular}{|c|c|c|c|c|c|}
\hline Method & $\begin{array}{c}\text { P1 } \\
(\mathrm{MW})\end{array}$ & $\begin{array}{c}\mathrm{P} 2 \\
(\mathrm{MW})\end{array}$ & $\begin{array}{c}\mathrm{P} 3 \\
(\mathrm{MW})\end{array}$ & $\begin{array}{c}\text { Losses } \\
(\mathrm{MW})\end{array}$ & $\begin{array}{c}\text { Optimum } \\
\text { Fuel Cost } \\
\$ / \mathrm{hr}\end{array}$ \\
\hline EP & 119.62 & 79.34 & 96.22 & 10.78 & 1186.9 \\
\hline PSO & 96.93 & 96.74 & 98.42 & 7.49 & 1199.3 \\
\hline HPSO & 129.42 & 66.27 & 96.20 & 8.28 & 1185.2 \\
\hline
\end{tabular}

\section{CONCLUSIONS}

The EP technique, PSO and Hybrid PSO algorithms were tested on IEEE 14 and IEEE 30 Bus systems and results were presented. The MVA line flow limits of the test systems were incorporated and the overload lines were observed. In the proposed HPSO method the performance of the PSO is greatly improved by incorporating EP features. The proposed method has been demonstrated to have superior features including stable convergence characteristics and avoid premature convergence. The proposed approach is relatively efficient, reliable and well suitable for large and practical utility systems.

\section{REFERENCES}

[1] Wood, A.J. Wollenberg, B.F.: "Power Generation Operation and Control" John Wiley and Sons, New York, 1984.

[2] Ringlee, R.J and Williams, D.D, 1963. Economic Dispatch operation considering valve throttling losses, II distribution of system loads by the method of dynamic programming, IEEE Transactions on Power Apparatus and Systems 82(1): 615622.

[3] Dommel, H.W. and Tinney, W.F, 1968 Optimal Power Flow Solutions, IEEE Transactions 87(10): 1866-1876

[4] Nanda, J, Hari, L and Kothari, M.L, 1994. Economic emission dispatch with line flow constraints using a classical technique, IEE proceedings on Generation, Transmission. Distribution 141(1): 1-10.

[5] Wong, K.P and Wong, Y.W 1995 Thermal Generator Scheduling Using Hybrid Genetic/ Simulated Annealing approach, IEE proceedings on Generation, Transmission. Distribution 142: 372-380.

[6] Desilva, I.N Nepomuceno, N. and Basdos, T.M, 2002. Designing a modified Hopfield network to solve an ED problem with non-linear cost function Proceedings of 
International Joint Conference on Neural Networks 2: 11601165.

[7] Baskar, G. Kumarappan, N. Mohan, M.R.: "Optimal Dispatch Using Improved Lambda Based Genetic Algorithm suitable for Utility System". International journal on Electric Power Components and Systems, June 2003.

[8] Nidul, S. Chakrabarti, R. Chattopadhyay, P.K.: "Evolutionary Programming Techniques for Economic Load Dispatch". IEEE Trans. on Evolutionary Computation, Feb. 2003.

[9] Zwe-Lee Gaing, "Particle Swarm Optimization to Solving ED Considering the Generator Constraints," IEEE Trans. Power syst., Vol.18 no.3, Aug2003 pp.1182-1195.

[10] Kennedy, J. Eberhart, R.: "Particle Swarm Optimization. Proc. of IEEE Int.Conf. on Neural Network". Perth Australia, 1995.

[11] Shi, Y. Eberhart, R.: "A Modified Particle Swarm Optimizer". Proc. of IEEE Int. Conf. on Evolutionary Computation, Anchorage, May 1998.

[12] Pancholi R.K., Swarup, K.S.: "Particle Swarm Optimization for Security Constrained Economic Dispatch". IEEE, Int. Conf. (ICISIP) 2004. 